MITSUBISHI ELECTRIC RESEARCH LABORATORIES

http://www.merl.com

\title{
On the Optimal Trajectory Generation for Servomotors: A Hamiltonian Approach
}

\author{
Wang, Y.; Ueda, K.; Bortoff, S.A.
}

TR2012-079 December 2012

\begin{abstract}
This note considers the energy optimal trajectory control design approach. Solving the exact optimal solution is challenging because of the nonlinear and switching cost function, and various constraints. The minimum principle is applied to establish piecewise necessary optimality conditions. An approximate optimal control is proposed to circumvent the difficulty due to the nonlinearity of the cost function. Simulation is performed to illustrate the generation of the approximate optimal trajectory.
\end{abstract}

IEEE Annual Conference on Decision and Control (CDC)

This work may not be copied or reproduced in whole or in part for any commercial purpose. Permission to copy in whole or in part without payment of fee is granted for nonprofit educational and research purposes provided that all such whole or partial copies include the following: a notice that such copying is by permission of Mitsubishi Electric Research Laboratories, Inc.; an acknowledgment of the authors and individual contributions to the work; and all applicable portions of the copyright notice. Copying, reproduction, or republishing for any other purpose shall require a license with payment of fee to Mitsubishi Electric Research Laboratories, Inc. All rights reserved. 



\title{
On the optimal trajectory generation for servomotors: a Hamiltonian approach
}

\author{
Yebin Wang, Koichiro Ueda, and Scott A. Bortoff
}

\begin{abstract}
This note considers the energy optimal trajectory generation of servo systems through open-loop optimal control design approach. Solving the exact optimal solution is challenging because of the nonlinear and switching cost function, and various constraints. The minimum principle is applied to establish piecewise necessary optimality conditions. An approximate optimal control is proposed to circumvent the difficulty due to the nonlinearity of the cost function. Simulation is performed to illustrate the generation of the approximate optimal trajectory.
\end{abstract}

\section{INTRODUCTION}

Reference trajectory generation plays a key role in the control of motion positioning systems using servomotors because the reference trajectory is identified as the main factor determining the performance of the resultant closedloop control system. A reference trajectory of a servomotor of a motion control system is in general generated by minimizing certain performance measures. Over decades, minimum time criteria has been widely used in reference trajectory generation to maximize productivity. A number of work have been reported on the time optimal or approximate time optimal trajectory generation, for instance, [9], [14], [11], [8], [16].

Another important criteria to generate a reference trajectory is the energy consumption of the motion control system. This is practically meaningful due to the fact that motor systems consume approximately $65 \%$ of the electricity in industry [19]. Existing work in this area includes the motor system steady state energy optimization [2], [4], energy-optimal control scheme for incremental motion drive (IMD) [15], [13], a heuristic approach [3], [18] as a few examples. Work [15], [13], [7], [3] did not address speed and acceleration constraints thus leads to conservativity in energy efficiency.

This note considers the energy optimal trajectory generation of servomotor systems through open-loop optimal control design approach. Speed and acceleration constraints are considered in the trajectory generation stage, and the optimal trajectory is to minimize the energy consumption of the motion control system including copper, amplifier, mechanical, and iron losses. The main difficulty in solving the optimal trajectory is result from the nonlinearity and switching in the cost function, and various constraints. This

Y. Wang, and S. A. Bortoff are with Mitsubishi Electric Research Laboratories, 201 Broadway, Cambridge, MA, 02139 USA. Tel:1-617-6217500; Fax: 1-617-621-7550; email: \{yebin.wang,bortoff\}@merl.com

K. Ueda is with the Advanced Technology R\&D Center, Mitsubishi Electric Corporation, 8-1-1, Tsukaguchi-honmachi, Amagasaki City, 6618661, Japan. email: Ueda.Koichiro@da.MitsubishiElectric.co.jp note derives piecewise necessary optimality conditions using the minimum principle.

This note is organized as follows. Section II introduces the problem. Main results are presented in Section III. Simulation is performed in Section IV to illustrate the generation of the approximate optimal trajectory.

\section{PRELIMINARY}

Consider the following second order servomotor model

$$
I \ddot{\theta}=K_{t} u-\bar{c}-\bar{d} \dot{\theta},
$$

where $\theta$ is the rotation angle of the motor, $I$ is the sum inertia of the load and servo motor, $K_{t}$ is the torque constant of the servo motor, $\bar{d}$ is the viscous friction coefficient, $\bar{c}$ is the Coulomb friction, and $u$ is the input current. The Coulomb friction usually changes its sign according to the velocity. The friction model used in this paper does not incorporates this because the considered optimal control problem includes a non-negative velocity constraint. The model is rewritten in the state space form

$$
\dot{x}=A x+B u+C,
$$

where $x=\left(x_{1}, x_{2}\right)^{T}=(\theta, \dot{\theta})^{T}$,

$$
A=\left[\begin{array}{cc}
0 & 1 \\
0 & -\frac{\bar{d}}{I}
\end{array}\right], \quad B=\left[\begin{array}{c}
0 \\
\frac{K_{t}}{I}
\end{array}\right], \quad C=\left[\begin{array}{c}
0 \\
\frac{-\bar{c}}{I}
\end{array}\right] .
$$

To simplify the presentation, we use notation: $d=\frac{\bar{d}}{I}, c=$ $\frac{\bar{c}}{I}, b=\frac{K_{t}}{I}$ in the sequel.

Note that the real servomotor dynamics is nonlinear from the saturation and hysteresis of the magnetic field, switches of amplifiers etc. Taking the Linear Time Invariant (LTI) model (1) is without loss of generality because the proposed methodology can be readily generalized to the nonlinear plant case.

\section{A. Loss Models}

A simple characterization of the energy consumption of a servo is its copper loss, which is consistent with the following quadratic cost function

$$
E=\int_{0}^{t_{f}} \frac{u^{2}}{2} \mathrm{~d} t
$$

The copper loss model (2) may not be valid for certain types of motors or certain positioning tasks, for instance, large servos or high speed tasks. This note considers the energy consumption model including the copper loss, the iron loss, 
the switching loss of amplifiers, and mechanical work, which is written as follows

$P(x, u)=R u^{2}+K_{e} x_{2}^{2} u^{2}+K_{h}\left|x_{2}\right||u|^{\gamma}+K_{s}|u|+K_{t} u x_{2}$,

where $R$ is the resistance of servo motor, $K_{e}$ and $K_{h}$ are constant coefficients of eddy current and hysteresis losses, $\gamma$ is constant, and $K_{s}$ is a constant coefficient of the switching loss. During the deceleration period, $P(x, u)$ could be negative. This means that the motor performs as a generator and converts mechanical work into electricity. The electricity however is not allowed to flow back to the utility grid. The energy consumption of a servo system is characterized by the following cost function

$$
\begin{aligned}
E & =\int_{0}^{t_{f}} Q(x(t), u(t)) \mathrm{d} t, \\
Q(x(t), u(t)) & = \begin{cases}P(x(t), u(t)), P(x(t), u(t))>0, \\
0, & P(x(t), u(t)) \leq 0,\end{cases}
\end{aligned}
$$

where $T$ is the tracking time.

\section{B. Problem Statement}

We shall compute the reference trajectory of a servo motor to minimize (3). The trajectory generation is treated as an open loop optimal control design problem as follows.

Problem 2.1: Given the plant (1), the initial state $x(0)=$ $x_{0}=(0,0)^{T}$, the final state $x\left(t_{f}\right)=x_{f}=(r, 0)^{T}$, and the final time $t_{f}$, find the control $u^{*}$ which minimizes certain cost function $E(u)$ subject to control, acceleration, and velocity constraints

$$
0 \leq x_{2} \leq v_{\max }, \quad\left|\dot{x}_{2}\right| \leq a_{\max }
$$

where $v_{\max }, a_{\max }, r$ are known constants.

Problem 2.1 with the cost function (2) has been studied intensively. For instance, by including the tracking error penalty in (2), the model predictive control has been applied and leads to a quadratic programming problem. Since both the cost function and the constraints are convex, the resultant numerical optimization problem has a global minimum. This property however does not hold for (3).

Numerous techniques have been proposed to treat inequality constraints, e.g. the integral penalty function approach considers the optimal control problem with a new cost which penalizes heavily along trajectory violating constraints. A more effective approach to solve such problem is to join together constrained and unconstrained arcs, making using of necessary optimality conditions. This approach is followed in the note to derive the necessary optimal conditions [1], [12], [6].

\section{Optimal CONTROL Trajectory Design}

Necessary conditions of an optimal solution to a state constrained optimal control problem have been investigated since 1960s. Readers are referred to [12], [1], [6], [5] for details. Sets of necessary optimality conditions can be obtained in various ways. For instance, [6] defines a Hamiltonian including the state constraint directly and establishes necessary conditions. We employ the approach which first converts a state constraint into a mixed state control constraint, then defines a Hamiltonian based on the resultant mixed state control constraint. For a state constraint, we introduce the following definition.

Definition 3.1: [1] The one dimensional state constraint $S(x) \leq 0$ has an order of $q$ if

$$
\begin{aligned}
S^{(k)}(x) & =0, \quad 0 \leq k \leq q-1, \\
S^{(q)}(x, u) & =0,
\end{aligned}
$$

where $S^{(k)}(x)$ is computed by differentiating $S(x) k$ times with respect to time.

To define a Hamiltonian, we first convert the velocity constraint into a mixed state control constraint

$$
\begin{array}{r}
x_{2}-v_{\max } \leq 0 \Rightarrow-d x_{2}-c+b u \leq 0, \\
-x_{2} \leq 0 \Rightarrow d x_{2}+c-b u \leq 0 .
\end{array}
$$

The acceleration constraint is a mixed state control constraint

$$
\begin{aligned}
& -d x_{2}-c+b u-a_{\max } \leq 0 \\
& -a_{\max }+d x_{2}+c-b u \leq 0 .
\end{aligned}
$$

\section{A. Treatment of the Switching Cost Function}

Given the switching nature of the cost functional and to simplify the presentation, we introduce notation of subtrajectories

$$
\begin{aligned}
& \mathcal{S}_{1}:\{t \mid P(t)>0\}, \quad \mathcal{S}_{1}=\mathcal{S}_{11} \cup \mathcal{S}_{12}, \\
& \mathcal{S}_{11}:\{t \mid P(t)>0, u(t)>0\}, \\
& \mathcal{S}_{12}:\{t \mid P(t)>0, u(t)<0\}, \\
& \mathcal{S}_{2}:\{t \mid P(t) \leq 0\},
\end{aligned}
$$

and rewrite the cost functional

$$
\begin{aligned}
E & =\int_{\mathcal{S}_{1}} P(t) \mathrm{d} t+\int_{\mathcal{S}_{2}} 0 \mathrm{~d} t \\
& =\int_{\mathcal{S}_{11}} P(t) \mathrm{d} t+\int_{\mathcal{S}_{12}} P(t) \mathrm{d} t+\int_{\mathcal{S}_{2}} 0 \mathrm{~d} t
\end{aligned}
$$

Remark 3.2: According to the optimality principle, a subtrajectory of a trajectory is also optimal. Therefore necessary conditions for each sub-trajectory within the sets $\mathcal{S}_{1}, \mathcal{S}_{2}$ to satisfy can be derived separately. The necessary conditions of the entire optimal trajectory can be established as a combination of necessary conditions of each sub-trajectory plus entry conditions between sub-trajectories.

Next we define a Hamiltonian over $\mathcal{S}_{1}, \mathcal{S}_{2}$ piecewisely, and attempt to derive necessary optimality conditions over different intervals sets $\mathcal{S}_{1}, \mathcal{S}_{2}$ from corresponding Hamiltonians. Notation: $H_{1}$ is the Hamiltonian over $\mathcal{S}_{1}, H_{11}$ and $H_{12}$ are the Hamiltonian over $\mathcal{S}_{11}, \mathcal{S}_{12}$ respectively, and $H_{2}$ is the Hamiltonian over $\mathcal{S}_{2}$.

\section{B. Necessary Optimality Conditions over Intervals $\mathcal{S}_{1}$}

Given $P(t)>0$ and the mixed state control constraints (5)-(6), we take the following Hamiltonian

$$
\begin{aligned}
H_{1}= & R u^{2}+K_{e} x_{2}^{2} u^{2}+K_{h}\left|x_{2}\right| \cdot|u|^{\gamma}+K_{s}|u| \\
& +K_{t} x_{2} u+\bar{H}_{1},
\end{aligned}
$$


where

$$
\begin{aligned}
\bar{H}_{1}= & \lambda^{T}(A x+B u+C)+\mu^{T}\left[\begin{array}{c}
-d x_{2}-c+b u \\
d x_{2}+c-b u
\end{array}\right] \\
& +\nu^{T}\left[\begin{array}{l}
-d x_{2}-c+b u-a_{\max } \\
-a_{\max }+d x_{2}+c-b u
\end{array}\right] .
\end{aligned}
$$

The Lagrange multipliers $\mu, \nu$ corresponding to the velocity and acceleration constraints, and satisfy sign conditions

$$
\begin{gathered}
\mu \begin{cases}=0, & \text { velocity constraint inactive, } \\
\geq 0, & \text { velocity constraint active, }\end{cases} \\
\nu \begin{cases}=0, & \text { acceleration constraint inactive, } \\
\geq 0, & \text { acceleration constraint active. }\end{cases}
\end{gathered}
$$

1) Optimal Control: Hamiltonian $H_{1}$ is not differentiable at $u=0$. We express $H_{1}$ piecewisely,

$$
H_{1}= \begin{cases}H_{11}=P_{1}+\bar{H}_{1}, & u>0, \\ H_{12}=P_{2}+\bar{H}_{1}, & u<0,\end{cases}
$$

where

$$
\begin{aligned}
& P_{1}=R u^{2}+K_{e} x_{2}^{2} u^{2}+K_{h} x_{2} u^{\gamma}+K_{s} u+K_{t} x_{2} u, \\
& P_{2}=R u^{2}+K_{e} x_{2}^{2} u^{2}+K_{h} x_{2}(-u)^{\gamma}-K_{s} u+K_{t} x_{2} u .
\end{aligned}
$$

We denote the positive control $u_{+}$over $\mathcal{S}_{1}$

$$
u_{+}=\arg \min _{P>0, u>0} H_{11},
$$

and the negative control $u_{-}$over $\mathcal{S}_{1}$

$$
u_{-}=\arg \min _{P>0, u<0} H_{12} \text {. }
$$

Proposition 3.3: Given $\gamma>1$ and $x_{2} \geq 0$, (9) has a unique solution $u_{+}$.

Proof: Since $u>0$ implies $P(x, u)>0$, (9) is equivalent to

$$
u_{+}=\arg \min _{0<u} H_{11} .
$$

Given $\gamma>1$, we further verify that the Legendre-Clebsch condition holds over $\mathcal{S}_{1}$,

$H_{11 u u}=\frac{\partial^{2} H_{11}}{\partial u^{2}}=2 R+2 K_{e} x_{2}^{2}+\gamma(\gamma-1) K_{h} x_{2} u^{\gamma-2}>0$.

Since the domain of the admissible control is convex, and $H_{11}$ is a convex function, we conclude the existence of the unique minimizer $u_{+}$.

We have a similar result about $u_{-}$.

Proposition 3.4: Given $\gamma>1$ and $x_{2} \geq 0$, (10) has a unique solution $u_{-}$.

Remark 3.5: Note that $u_{+}$cannot always be solved from the first order necessary condition

$$
H_{11 u}=\frac{\partial H_{11}}{\partial u}=0 .
$$

Neither can $u_{-}$necessarily be solved from

$$
H_{12 u}=\frac{\partial H_{12}}{\partial u}=0 .
$$

Propositions 3.3 and 3.4 however establish the uniqueness of solutions of (9) and (10), which is important to show the sufficiency of necessary optimality conditions. Also, Propositions 3.3 and 3.4 guarantee the solvability of the unique control $u_{+}$and $u_{-}$through numerical optimization approach [10].

Assuming that at any time instant, only one constraint is active, then the control on constrained arcs is readily obtained as follows

$$
u=\left\{\begin{array}{l}
\frac{d x_{2}+c}{b}, \quad \text { velocity constraint is active, } \\
\frac{a_{\max }+d x_{2}+c}{b}, \quad \dot{x}_{2}-a_{\max } \leq 0 \text { is active, } \\
\frac{-a_{\max }+d x_{2}+c}{b}, \quad-a_{\max }-\dot{x}_{2} \leq 0 \text { is active. }
\end{array}\right.
$$

2) Costate Dynamics: Since $x_{2} \geq 0$, the partial derivative of $H_{1}$ w.r.t. $x$ is well-defined, the costate dynamics can be readily obtained.

$$
\begin{aligned}
\dot{\lambda}= & -A^{T} \lambda-\left[2 K_{e} x_{2} u^{2}+K_{h}|u|^{\gamma}+K_{t} u\right] \\
& -\left[\begin{array}{cc}
0 & 0 \\
-d & d
\end{array}\right](\mu+\nu) .
\end{aligned}
$$

When the system is along unconstrained arcs, we have $\mu=$ $\nu=0$. The costate is continuous at the entry point of the unconstrained trajectory. For the constrained arcs, we need to determine $\mu, \nu$ and the boundary conditions of $\lambda$ at their entry points. We know the jumps of costate arise from interior points conditions. The acceleration constraint therefore will not incur jumps of the costate during the entry of constrained arcs. The corresponding costate dynamics is

$$
\begin{aligned}
& \dot{\lambda}=-A^{T} \lambda-\left[\begin{array}{c}
0 \\
2 K_{e} x_{2} u^{2}+K_{h}|u|^{\gamma}+K_{t} u
\end{array}\right]-\left[\begin{array}{cc}
0 & 0 \\
-d & d
\end{array}\right] \nu, \\
& \lambda\left(t_{\text {entry }}^{+}\right)=\lambda\left(t_{\text {entry }}^{-}\right),
\end{aligned}
$$

where $\nu$ is solved from

$$
\begin{aligned}
& \left.H_{1 u}\right|_{u=\frac{a_{\max +d x_{2}+c}>0}{b}=0, \quad \dot{x}_{2}-a_{\max } \leq 0 \text { is active, }}=0, \quad-a_{\max }-\dot{x}_{2} \leq 0 \text { is active. } \\
& \left.H_{1 u}\right|_{u=\frac{-a_{\max }+d x_{2}+c}{b}<0}=0 .
\end{aligned}
$$

Equation (13a) has a solution

$$
\begin{aligned}
\nu_{1}= & \frac{-1}{b}\left\{2 R u+2 K_{e} x_{2}^{2} u+\gamma K_{h} x_{2} u^{\gamma-1}\right. \\
& \left.+K_{s}+K_{t} x_{2}+b \lambda_{2}\right\} .
\end{aligned}
$$

To ensure $\nu_{1} \geq 0, \lambda_{2}$ should be negative. Similarly, (13b) has a solution

$$
\begin{aligned}
\nu_{2}= & \frac{1}{b}\left\{2 R u+2 K_{e} x_{2}^{2} u-\gamma K_{h} x_{2}(-u)^{\gamma-1}\right. \\
& \left.-K_{s}+K_{t} x_{2}+b \lambda_{2}\right\} .
\end{aligned}
$$

On the other hand, the velocity constraint may incur jumps of the costate. Since the velocity constraint is one order state constraint, it will not become active as a touch point, i.e., we only need to consider the case when the velocity constraint is active over arcs. For the constraint $x_{2}-v_{\max } \leq 0$, denoting the interior point constraint

$$
N_{1}=x_{2}-v_{\max }
$$


we have the boundary condition at the entry point of a velocity constrained arc

$$
\begin{aligned}
\lambda\left(t_{\text {entry }}^{+}\right) & =\lambda\left(t_{\text {entry }}^{-}\right)-\pi_{1}\left(\frac{\partial N}{\partial x}\right)^{T}\left(t_{\text {entry }}^{-}\right) \\
& =\lambda\left(t_{\text {entry }}^{-}\right)-\pi_{1}\left[\begin{array}{l}
0 \\
1
\end{array}\right], \\
H\left(t_{\text {entry }}^{+}\right) & =H\left(t_{\text {entry }}^{-}\right)+\pi_{1} \frac{\partial N}{\partial t}\left(t_{\text {entry }}^{-}\right),
\end{aligned}
$$

where $\pi_{1} \in \mathbb{R}$ is a Lagrange multiplier. Similarly, for the constraint $-x_{2} \leq 0$,

$$
N_{2}=-x_{2},
$$

the jump conditions are

$$
\begin{aligned}
\lambda\left(t_{\text {entry }}^{+}\right) & =\lambda\left(t_{\text {entry }}^{-}\right)-\pi_{2}\left[\begin{array}{c}
0 \\
-1
\end{array}\right], \\
H\left(t_{\text {entry }}^{+}\right) & =H\left(t_{\text {entry }}^{-}\right) .
\end{aligned}
$$

The costate dynamics is therefore rewritten as

$$
\begin{aligned}
& \dot{\lambda}=-A^{T} \lambda-\left[\begin{array}{c}
0 \\
2 K_{e} x_{2} u^{2}+K_{h}|u|^{\gamma}+K_{t} u
\end{array}\right]-\left[\begin{array}{cc}
0 & 0 \\
-d & d
\end{array}\right] \mu, \\
& \lambda\left(t_{\text {entry }}^{+}\right)=\lambda\left(t_{\text {entry }}^{-}\right)-\left[\begin{array}{c}
0 \\
\pi_{1}
\end{array}\right], \quad x_{2}-v_{\max } \leq 0 \text { is active, } \\
& \lambda\left(t_{\text {entry }}^{+}\right)=\lambda\left(t_{\text {entry }}^{-}\right)+\left[\begin{array}{c}
0 \\
\pi_{2}
\end{array}\right], \quad-x_{2} \leq 0 \text { is active }
\end{aligned}
$$

The Lagrange multiplier $\mu$ is determined from $H_{1 u}=0$ with $u=\left(d x_{2}+c\right) / b$. Different from the acceleration constraint, $u>0$ when the velocity constraint is active. We use $H_{11}$ to compute $H_{1 u}$ and solve

$$
\begin{aligned}
\mu_{1}= & \frac{-1}{b}\left\{2 R u+2 K_{e} x_{2}^{2} u+\gamma K_{h} x_{2} u^{\gamma-1}\right. \\
& \left.+K_{s}+K_{t} x_{2}+b \lambda_{2}\right\}, \quad x_{2}=v_{\max } \\
\mu_{2}= & \frac{-1}{b}\left\{2 R u+K_{s}+b \lambda_{2}\right\}, \quad x_{2}=0,
\end{aligned}
$$

where $u=\left(d x_{2}+c\right) / b$.

Remark 3.6: Notice when the velocity constraint is active, $u=\left(d x_{2}+c\right) / b>0$ and $P(x, u)>0$. Hence, the velocity constrained arcs always belong to $\mathcal{S}_{1}$. Similarly, the arcs where the positive acceleration constraint $\dot{x}_{2} \leq a_{\max }$ is active belong to $\mathcal{S}_{1}$ too. For the negative acceleration constraint $-\dot{x}_{2} \leq a_{\max }, u=\left(-a_{\max }+d x_{2}+c\right) / b$ is generally negative, which may render $P(x, u)<0$.

\section{Necessary Optimality Conditions over Intervals $\mathcal{S}_{2}$}

Interval set $\mathcal{S}_{2}$ is characterized by the constraint $P(x, u) \leq$ 0 which requires $u \leq 0$. According to Remark 3.6, over intervals $\mathcal{S}_{2}$, the Hamiltonian $H_{2}=\bar{H}_{1}$ except $\mu=0, \nu_{1}=$ 0 , i.e.,

$$
H_{2}=\lambda^{T}(A x+B u+C)+\nu_{2}\left(-a_{\max }+d x_{2}+c-b u\right),
$$

which is differentiable w.r.t. $x$ and $u$. The corresponding costate dynamics is

$$
\begin{aligned}
& \dot{\lambda}_{1}=0, \\
& \dot{\lambda}_{2}=-\lambda_{1}+d \lambda_{2}-d \nu_{2} .
\end{aligned}
$$

Assuming the negative acceleration constraint is active over $\mathcal{S}_{2}$, we have

$$
\frac{\partial H_{2}}{\partial u}=b \lambda_{2}-b \nu_{2}=0,
$$

and solve $\nu_{2}=\lambda_{2}$. The corresponding $\lambda_{2}$ dynamics is given by $\dot{\lambda}_{2}=-\lambda_{1}$. Note that the sign condition of $\nu_{2}$ requires $\lambda_{2} \geq 0$ when the negative acceleration constraint is active over $\mathcal{S}_{2}$.

Over the unconstrained arcs, $\nu_{2}=0$, thus $H_{2}$ can be written as

$$
H_{2}=\bar{H}_{2}(x, \lambda)+\lambda^{T} B u=\bar{H}_{2}(x, \lambda)+b \lambda_{2} u .
$$

It is clear that if $\lambda_{2} \neq 0$, the optimal control is in the form of Bang-Bang. Otherwise, we have a singular control problem.

Remark 3.7: We use contradiction to show the optimal trajectory does not include a singular arc. Assume a singular arc exists. Because the optimal control is uniquely defined over constrained arcs, we only need to consider the unconstrained arcs. Hence the costate dynamics is

$$
\dot{\lambda}=-A^{T} \lambda \text {. }
$$

The fact that $\lambda_{2} \equiv 0$ over a singular arc and (18) implies $\lambda_{1} \equiv 0$. Since $\dot{\lambda}_{1} \equiv 0$, we know $\lambda_{1} \equiv 0$ over $\left[0, t_{f}\right]$, and $\dot{\lambda}_{2}=d \lambda_{2}$. According to the continuity of optimal control, a positive unconstrained control arc is always prior to the zero control arc. This implies the costate $\lambda_{2}\left(t_{1}\right)<0$ with $t_{1}$ denoting the entry time of the zero control arc. Given a negative initial condition, the costate $\lambda_{2}$ is monotonically decreasing and cannot reach zero. This contradicts the fact $\lambda_{2} \equiv 0$.

We consider the case when $\lambda_{2}=0$ at finite points. Note that $P(x, u) \leq 0$ allows a larger domain of admissible control than $P(x, u) \leq-\epsilon<0$. Hence, the control over unconstrained arcs should be solved from

$$
\arg \min _{u} H_{2} \quad \text { subject to } P(x, u) \leq 0 .
$$

It can be shown that given $\gamma>1, x_{2} \geq 0$, the inequality $P(x, u) \leq 0$ gives a convex domain $D \subset \mathbb{R}^{-} \cup\{0\}$. Since $H_{2}$ is a linear function of $u$, (19) has a unique minimizer $u_{0}$. Given the domain $D$ and the sign of $\lambda_{2}$, we have the control

$$
u_{0}=\left\{\begin{array}{l}
\min \{D\}, \quad \lambda_{2}>0, \\
\max \{D\}=0, \quad \lambda_{2}<0 .
\end{array}\right.
$$

Remark 3.8: To simplify the computation of domain $D$, we assume $\gamma=1$ and have a non-trivial approximate solution of $P(x, u)=0$ as follows

$$
u_{3}=\frac{\left(K_{h}-K_{t}\right) x_{2}+K_{s}}{R+K_{e} x_{2}^{2}} \text { s.t. } P\left(x, u_{3}\right) \approx 0 .
$$

Since $K_{h}<K_{t}, u_{3}<0$ when $x_{2}>\frac{K_{s}}{K_{t}-K_{h}}=x_{2}^{B}$. We therefore have the domain of $u$ satisfying $P(x, u) \leq 0$

$$
D:\left\{\begin{array}{l}
\{0\}, \quad x_{2} \leq x_{2}^{B}, \\
u_{3} \leq u \leq 0, \quad x_{2} \geq x_{2}^{B} .
\end{array}\right.
$$


Given the domain $D$ in (22), we have the approximate solution of (19)

$$
u_{0}=\left\{\begin{array}{l}
0, \quad x_{2} \leq x_{2}^{B}, \\
\min \{D\}=u_{3}, \quad x_{2} \geq x_{2}^{B} \text { and } \lambda_{2}>0 \\
\max \{D\}=0, \quad x_{2} \geq x_{2}^{B} \text { and } \lambda_{2}<0
\end{array}\right.
$$

\section{Entry Boundary Conditions}

The piecewise Hamiltonian implies that the optimal trajectory might switch. We need to derive the boundary conditions rising from switches among sub-trajectories $\mathcal{S}_{11}, \mathcal{S}_{12}, \mathcal{S}_{2}$. These conditions are referred as the Weierstrass-Erdmann corner conditions. We exemplify the necessary conditions for the switch from $\mathcal{S}_{11}$ to $\mathcal{S}_{12}$. Without loss of generality, we assume the switch happens at $t_{1}$ and have the boundary condition at the switch point

$$
\begin{aligned}
H_{11}\left(t_{1}^{-}\right) & =H_{12}\left(t_{1}^{+}\right), \\
\lambda\left(t_{1}^{-}\right) & =\lambda\left(t_{1}^{+}\right) .
\end{aligned}
$$

Combining with the continuity of state, (24) is equivalent to

$$
\begin{aligned}
P_{1}\left(t_{1}^{-}\right)+b \lambda_{2}\left(t_{1}^{-}\right) u\left(t_{1}^{-}\right) & =P_{2}\left(t_{1}^{+}\right)+b \lambda_{2}\left(t_{1}^{+}\right) u\left(t_{1}^{+}\right), \\
\lambda\left(t_{1}^{-}\right) & =\lambda\left(t_{1}^{+}\right) .
\end{aligned}
$$

The first boundary condition actually determines the switch time $t_{1}$. Conditions of switches for other cases can be similarly obtained. These conditions also means the piecewise Hamiltonian is continuous along the optimal trajectory of Problem 2.1.

\section{Computation of Sub-optimal Trajectories}

A number of direct computation methods have been proposed and applied to solved constrained optimal control problems. The main idea of direct computation is to transcript the optimal control design into a nonlinear programming (NLP) problem over finite dimensional parameter space. An NLP solver is used to solve the resultant NLP problem. Compared with the indirect approach, e.g. solving the optimal control trajectory from necessary conditions, direct computation has advantages on capabilities to handle complicated constraints and performance metrics. It however suffers from issues such as convergence speed. Readers can refer to [17] and references therein for detailed review on direction computation. This note relies on the aforementioned necessary conditions to construct the optimal trajectory.

\section{A. Indirect Approach}

As shown in Section III, necessary conditions are written as a set of ordinary differential equations (ODEs) and nonlinear algebraic equations (NAEs) which are piecewisely defined over different time intervals. ODEs defines the dynamics of state and costate. NAEs defines the boundary conditions, which includes the initial and final state conditions, terminal conditions of each arc, terminal conditions of each sub-trajectories, jump conditions on costate, conditions on Lagrange multipliers, switch conditions over arcs etc.
Therefore, necessary conditions can be formulated a series of Multi-Point Boundary Value Problems (MBVPs). Solving an MBVP generally requires the knowledge of the structure of the optimal trajectory, which could be obtained through analysis or iterative programming procedure. Assuming the knowledge of the structure of the optimal trajectory, the MBVP to be solved is well-defined.

\section{B. Simulation}

Given the MBVP, and using Matlab function $b v p 5 c$, we have the simulation results shown in Figures 1-4. As shown in Figures 1-2, the optimal trajectory for one case of problem data has 5 arcs: positive acceleration constrained arc, positive unconstrained arc, zero control and zero power arc, negative unconstrained arc, and negative acceleration constrained arc. The negative unconstrained and non-positive power arc, which corresponds to $u_{3}$, does not appears because $x_{2}<x_{2}^{B}$. Figures 3-4 show that the optimal trajectory for another case of problem data includes 6 arcs. The Hamiltonian is not constant over the trajectory while $u_{3}$ is applied. This is because $u_{3}$ is an approximate solution of $P(x, u)=0$, and the numerical solver hasn't converged to the exact solution yet. In fact, Hamiltonian should always be constant along the optimal trajectory of Problem 2.1. For both cases, the optimal trajectories try to maintain zero power consumption as longer as possible during the deceleration period.
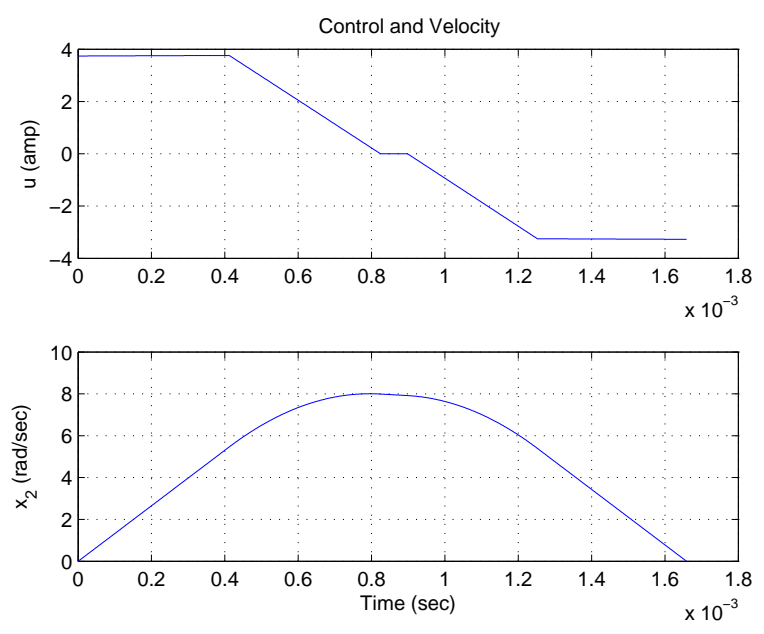

Fig. 1. Case 1: the trajectories of control and velocity

\section{CONCLUSION}

This paper discussed the energy optimal trajectory generation of servo systems in the open-loop optimal control design framework. Due to the switching cost function, piecewise necessary optimality conditions were resulted and derived. Simulation illustrates the generation of the approximate optimal trajectory.

\section{REFERENCES}

[1] A. E. Bryson, Jr. and Y.-C. Ho, Applied Optimal Control: Optimization, Estimation, and Control. NY: Taylor \& Francis Group, 1975. 

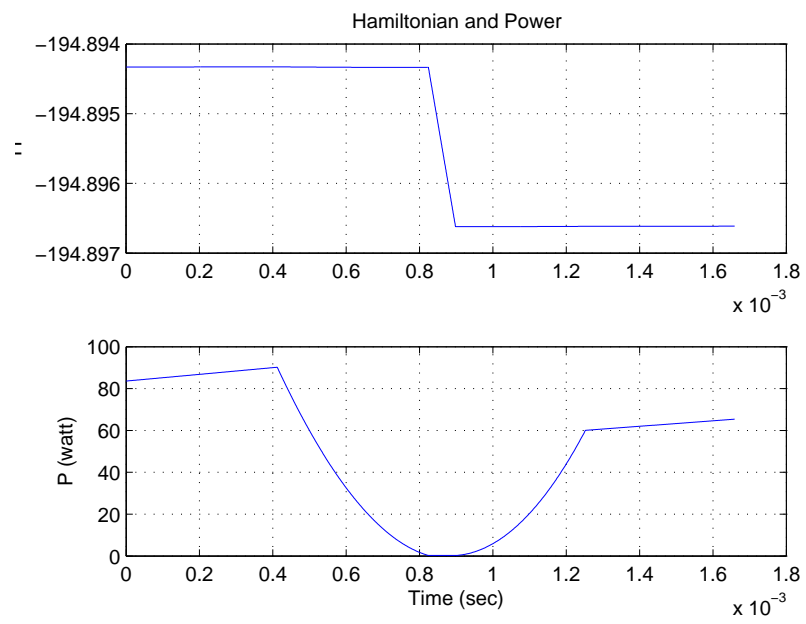

Fig. 2. Case 1: the trajectories of Hamiltonian and power
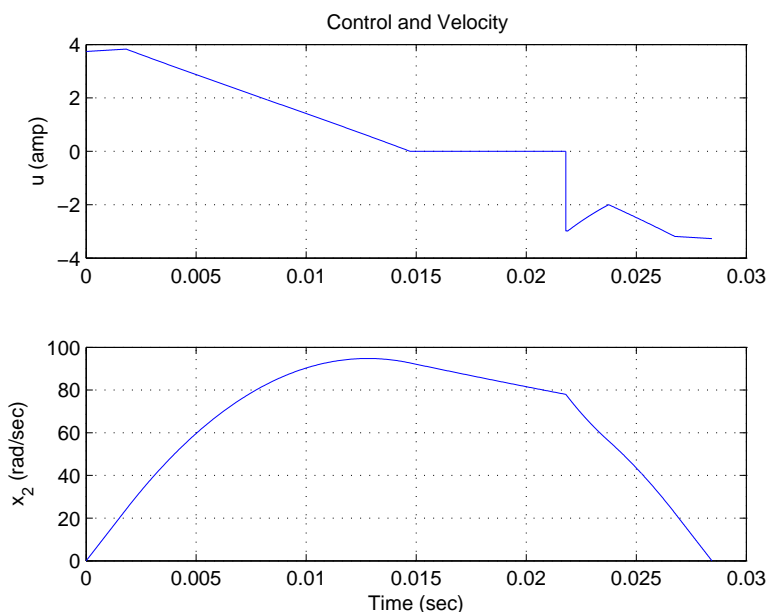

Fig. 3. Case 2: the trajectories of control and velocity
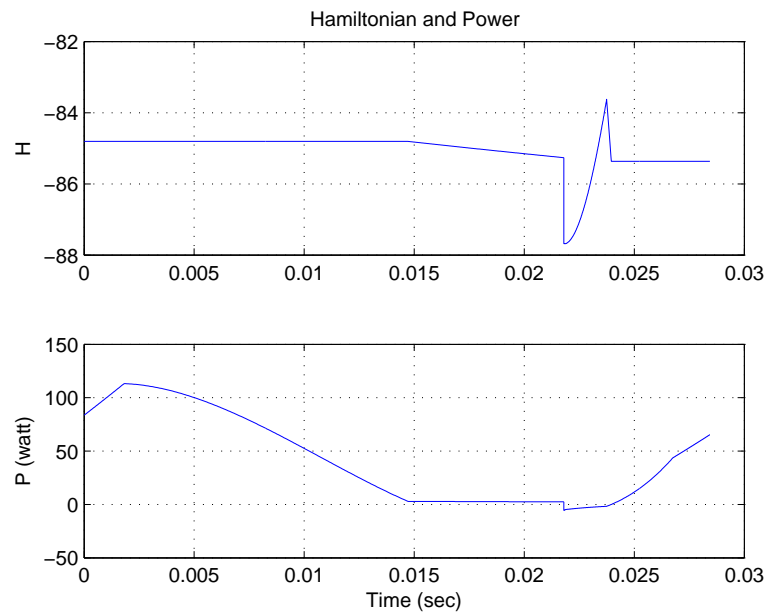

Fig. 4. Case 2: the trajectories of Hamiltonian and power

[2] F. Abrahamsen, F. Blaabjerg, J. Pedersen, P. Grabowski, and P. Thogersen, "On the energy optimized control of standard and high-efficiency induction motors in ct and hvac applications," IEEE Transactions on Industry Applications, vol. 34, no. 4, pp. 822 -831, Jul/Aug 1998.

[3] S. Dodds, "Sliding mode vector control of pmsm drives with minimum energy position following," in 13th Power Electronics and Motion Control Conference, 2008., Sep. 2008, pp. 2559-2566.

[4] S. Ghozzi, K. Jelassi, and X. Roboam, "Energy optimization of induction motor drives," in IEEE International Conference on Industrial Technology, vol. 2, Dec. 2004, pp. $602-610$.

[5] R. F. Hartl, S. P. Sethi, and R. G. Vickson, "A survey of the maximum principles for optimal control problems with state constraints," SIAM Review, vol. 37, pp. 181-218, Jun. 1995.

[6] D. H. Jacobson, M. M. Lele, and J. L. Speyer, "New necessary conditions of optimality for control problems with state-variable inequality constraints," Journal of Mathematical Analysis and Application, vol. 35, pp. 255-284, 1971.

[7] C. H. Kim and B. K. Kim, "Minimum-energy translational trajectory generation for differential-driven wheeled mobile robots," Journal of Intelligent \& Robotic Systems, vol. 49, no. 4, pp. 367-383, 2007.

[8] C. La-orpacharapan and L. Y. Pao, "Shaped time-optimal feedback control for disk-drive systems with back-electromotive force," IEEE Transaction on Magnetics, vol. 40, no. 1, pp. 85-96, 2004.

[9] P. Lambrechts, M. Boerlage, and M. Steinbuch, "Trajectory planning and feedforward design for electromechnical motion systems," Control Engineering Practice, vol. 13, pp. 145-157, Jan. 2005.

[10] J. Nocedal and S. J. Wright, Numerical Optimization. Springer, 2006.

[11] M.-H. Park and C.-Y. Won, "Time optimal control for induction motor servo system," IEEE Transaction on Power Electronics, vol. 6, no. 3, pp. 514-524, July 1991.

[12] H. J. Pesch, "A practical guide to the solution of real-life optimal control problems," Control and Cybernetics, vol. 23, pp. 7-60, 1994

[13] M. A. Sheta, V. Agarwal, and P. S. V. Nataraj, "A new energy optimal control scheme for a separately excited dc motor based incremental motion drive," International Journal of Automation and Computing, vol. 6, no. 3, pp. 267-276, 2009.

[14] G. Toacse and W. Culpi, "Time-optimal control of a stepping motor," IEEE Transactions on Industrial Electronics and Control Instrumentation, vol. IECI-23, no. 3, pp. 291 -295, Aug. 1976.

[15] A. Trzynadlowski, "Energy optimization of a certain class of incremental motion dc drives," IEEE Transactions on Industrial Electronics, vol. 35, no. 1, pp. 60-66, Feb 1988.

[16] M. Vasak, M. Baotic, I. Petrovic, and N. Peric, "Hybrid theory-based time-optimal control of an electronic throttle," IEEE Transactions on Industrial Electronics, vol. 54, no. 3, pp. 1483 -1494, Jun. 2007.

[17] D. Verscheure, B. Demeulenaere, J. Swevers, J. D. Schutter, and M. Diehl, "Time-optimal path tracking for robots: A convex optimization approach," IEEE Trans. Automat. Contr., vol. AC-54, no. 10, pp. 2318-2327, Oct. 2009.

[18] J. Vittek, P. Bris, I. Skalka, R. Filka, P. Minarech, and J. Faber, "Experimental verification of energy saving position control algorithm applied to the drives with pmsm," in 2010 14th International Power Electronics and Motion Control Conference (EPE/PEMC), Sep. 2010 pp. S10-1 -S10-6.

[19] E. Worrell, L. Bernstein, J. Roy, L. Price, and J. Harnisch, "Industrial energy efficiency and climate change mitigation," Energy Efficiency, vol. 2, no. 2, pp. 109-123, 2009. 